\title{
Metabolomic Profiling of the Aqueous Humor in Patients with Wet Age-Related Macular Degeneration using UHPLC-MS/MS
}

\author{
Supporting Information
}

\author{
Guoge Han ${ }^{1, *}$, Pinghui Wei ${ }^{1}$, Meiqin He ${ }^{1}$, He Teng ${ }^{2}$,Yanhua Chu ${ }^{1}$ \\ ${ }^{1}$ Tianjin Eye Hospital, Tianjin Key Lab of Ophthalmology and Visual Science, Tianjin, P.R. China \\ ${ }^{2}$ Eye Institute and School of Optometry and Ophthalmology, Tianjin Medical University Eye \\ Hospital, Tianjin, P.R. China \\ * Correspondence to: Guoge Han, Tianjin Eye Hospital, Tianjin Key Lab of Ophthalmology and \\ Visual Science, Tianjin, 300020, P.R. China; dovehanguoge@hotmail.com
}

\section{Supporting Information File 1}

Figure S1. Score plots of principal components analysis (PCA) from wet AMD and control groups. $\mathrm{t}[1]=$ first principal component; $\mathrm{t}[2]=$ second principal component .

Figure S2. Heatmap representation of 18 metabolites across 35 samples. Each line in the heat map represented a metabolite. The deeper the red color, the higher its content in the tested sample; similarly, the deeper the blue color, the lower its content in the tested sample.

Figure S3. Spearman correlation analysis of all the significant change metabolites (VIP $>1$ and $\mathrm{p}<0.05)$.

\section{Supporting Information File 2}

Excel document of all detected metabolites. 


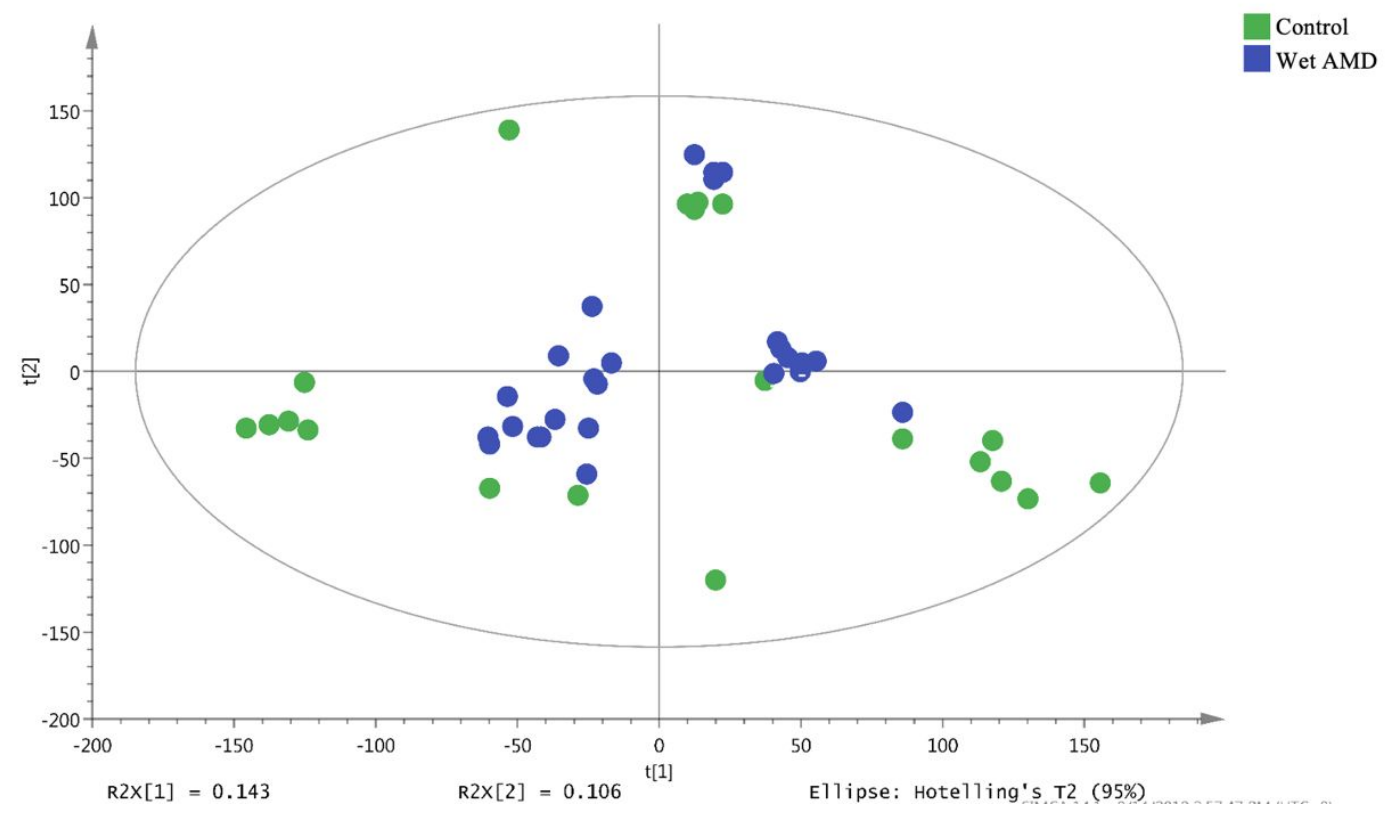

Figure S1. Score plots of principal components analysis (PCA) from wet AMD and control groups. $\mathrm{t}[1]=$ first principal component; $\mathrm{t}[2]=$ second principal component . 


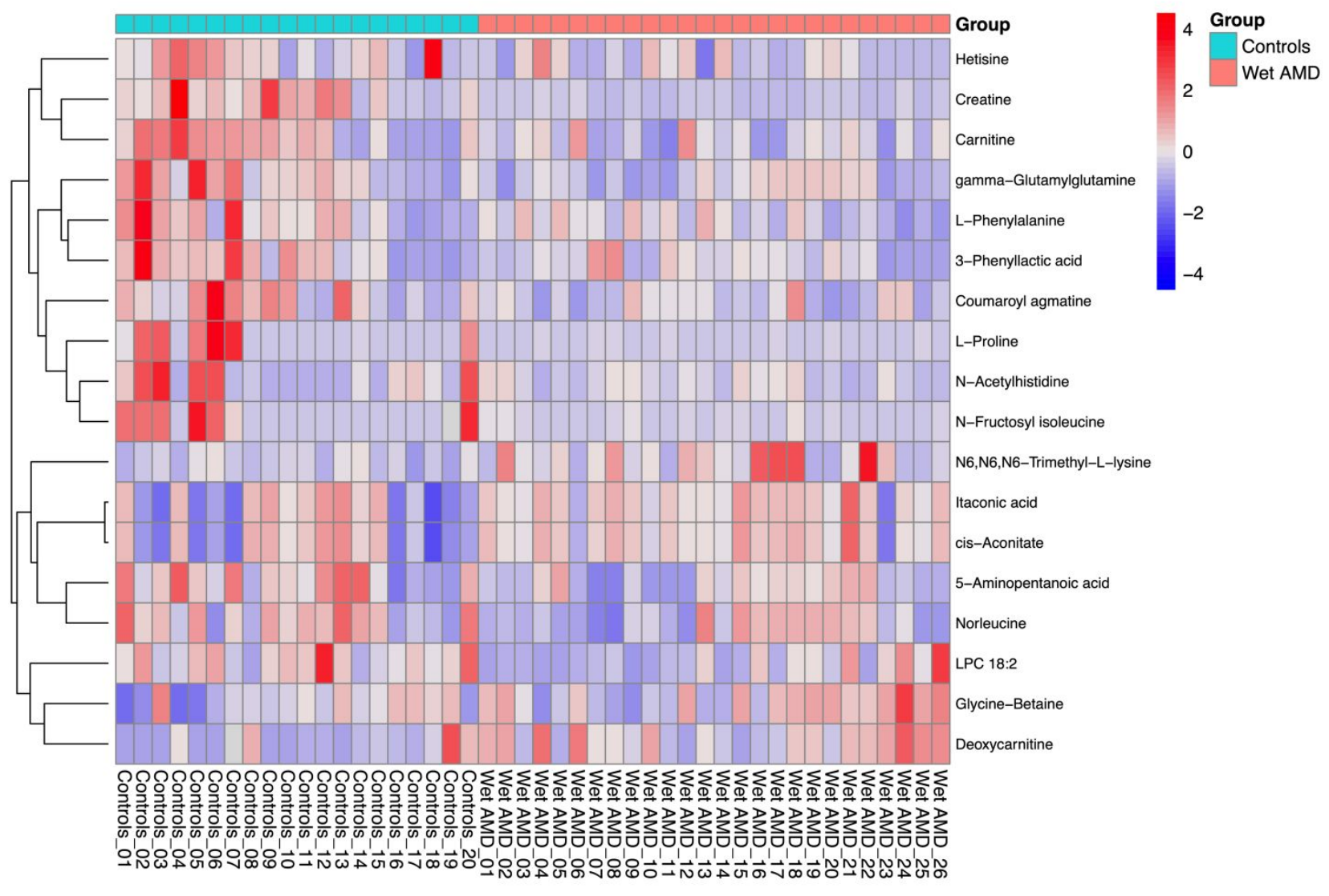

Figure S2. Heatmap representation of 18 metabolites across 35 samples. Each line in the heat map represented a metabolite. The deeper the red color, the higher its content in the tested sample; similarly, the deeper the blue color, the lower its content in the tested sample. 


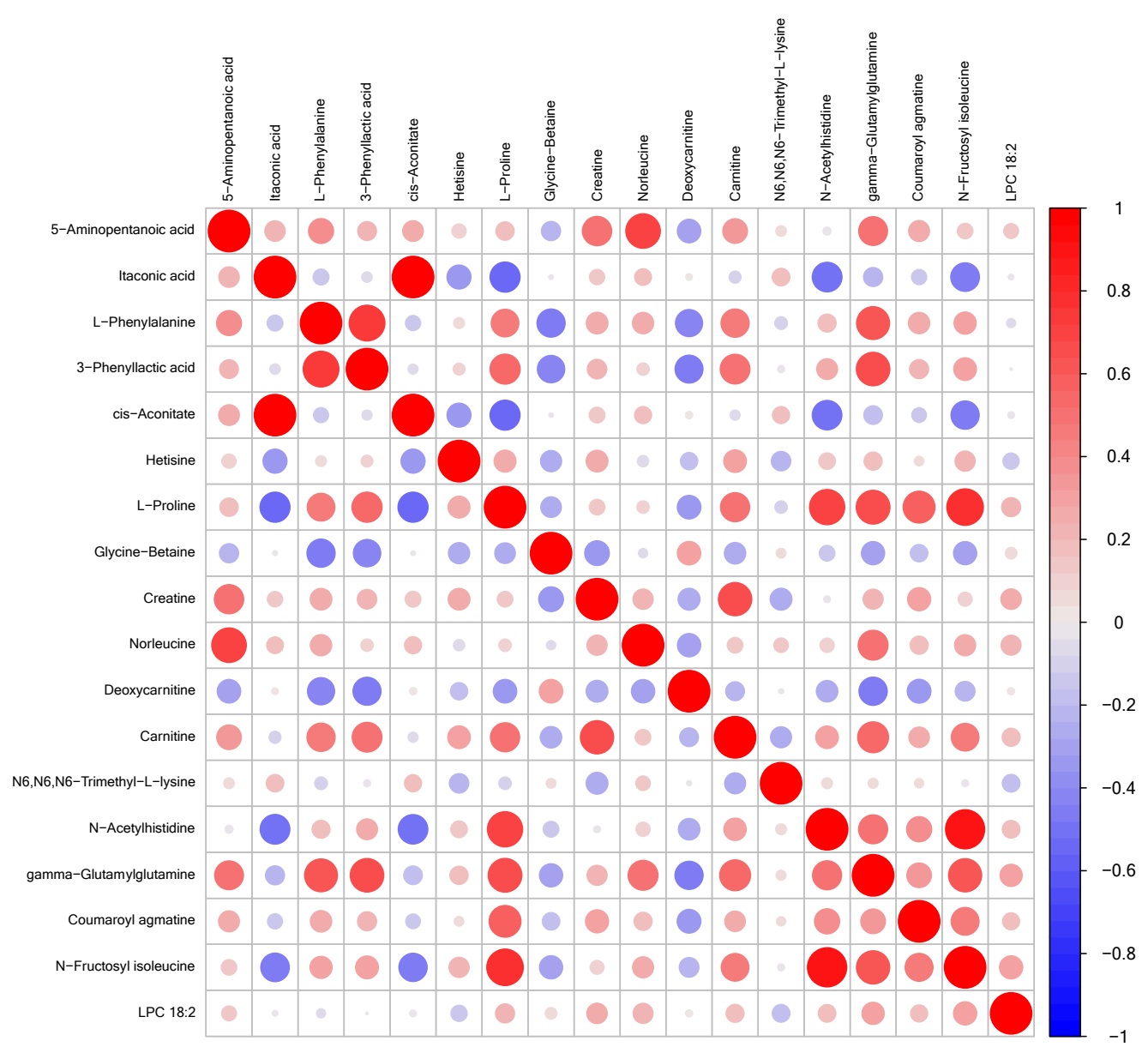

Figure S3. Spearman correlation analysis of all the significant change metabolites (VIP $>1$ and $\mathrm{p}<0.05)$. 